\title{
PRIMARY MODULES DETERMINED BY INDECOMPOSABLE IDEMPOTENT ENDOMORPHISMS
}

\author{
ROBERT W. STRINGALL
}

\begin{abstract}
A faithful primary module over a complete discrete valuation ring is determined up to isomorphism by any subring of the endomorphism ring of the module which contains all the indecomposable indempotent endomorphisms.
\end{abstract}

This note is part of a study centered on the problem of ascertaining to what extent the decompositions of a primary Abelian group determine the group. Perhaps the most useful interpretation of this problem lies in the connection between direct summands of a group and idempotent elements in the endomorphism ring of the group. One of the advantages of this viewpoint is that it facilitates the introduction of Boolean structures into the general theory of Abelian p-groups (see, for example, [5]). This becomes important once consideration is given to the state of the search for invariants for primary Abelian groups. In short, there seems to be no other recourse but to redefine, in terms of structural acceptability, what the invariants may be.

It is the main objective of this note to show that there is some basis for the belief that the theory of Boolean rings can serve as an important vehicle for the characterization of Abelian p-groups.

I. Kaplansky has shown that faithful primary modules over complete discrete valuation rings are determined up to isomorphism by their endomorphism rings ([2, Theorem 28]). The fact that there are proper subrings of the endomorphism ring which determine the module was established by R. S. Pierce (see [4, 12.2 and 3.9]). Using the definition of indecomposable idempotent found in [3, p. 132], and employing some modifications, Kaplansky's proof can easily be extended to validate the following theorem.

THEOREM. Let $R$ be a complete discrete valuation ring, $M$ and $N$ faithful primary $R$-modules. If $E$ and $F$ are, respectively, $R$-subrings of the

Received by the editors December 15, 1970 and, in revised form, March 8, 1971. AMS 1970 subject classifications. Primary 20K10, 20K25; Secondary 20K30.

Key words and phrases. Faithful primary modules over complete discrete valuation rings, subring generated by idempotent endomorphisms, subring generated by automorphisms. 
endomorphism rings of $M$ and $N$ containing the indecomposable idempotent endomorphisms of $M$ and $N$, then any $R$-isomorphism of $E$ and $F$ is induced by isomorphism of $M$ and $N$.

Proof. Accepting the proof of [2, Theorem 28], in notation and form, it suffices to check that the endomorphisms constructed can be taken in $E$ and $F$. As a sample of the details, it will be shown that in case II there are suitable maps $C$ in $E$ "sending $x$ to $t_{2}$ and $x_{i}$ (for $i$ sufficiently large) into $t_{1}$, and annihilating the complementary summands." First note that the construction of a map $C^{\prime} \in E$ such that $C^{\prime}$ sends $x$ to $t_{2}$ and annihilates $M(1-e)$ is not difficult and is in fact similar to a portion of what follows. To complete the argument, it will be shown that for $i$ large enough, there exists a map $C_{i} \in E$ which carries $x_{i}$ to $t_{1}$ and which annihilates $M\left(1-e_{1}\right)$. To do this write $t_{1}=t_{1} e_{1}+t_{1}\left(1-e_{1}\right)$. Clearly, there exists a least $i_{0}$ such that $t_{1} e_{1}=x_{i_{0}} \beta_{i_{0}} e_{1}$ for some $\beta_{i_{0}}$ in $R$. If $i \geqq i_{0}$, let $\hat{C}_{i}=p^{i-i_{0}} \beta_{i_{0}} e_{1}$. Then $x_{i} \hat{C}_{i}=p^{i-i_{0}} x_{i} \beta_{i_{0}} e_{1}=x_{i_{0}} \beta_{i_{0}} e_{1}=t_{1} \dot{e}_{1}$. If $t_{1}\left(1-e_{1}\right)=$ 0 , let $C_{i}=\hat{C}_{i}$. Otherwise, let $K$ be a rank one summand of $M(1-e)$ which contains $t_{1}\left(1-e_{1}\right)$. Let $k_{1}, k_{2}, \cdots$ be a generating set for $K$ with the standard relations $p^{i} k_{i+j}=k_{j}(i+j \geqq 1)$ and assume for some $j, k_{j}=$ $t_{1}\left(1-e_{1}\right)$. Let $\hat{C}$ be the endomorphism of $M$ sending $x_{i}$ to $x_{i}+k_{i}$ for all $i$ and annihilating $M\left(1-e_{1}\right)$. Then $\hat{C}$ is an indecomposable idempotent. For $i \geqq j, i_{0}$, let $C_{i}=p^{i-j} \hat{C}-p^{i-j} e_{1}+p^{i-i_{0}} \beta_{i_{0}} e_{1}$. Then $C_{i} \in E$ and

$$
\begin{aligned}
x_{i} C_{i} & =p^{i-j} x_{i} \hat{C}-p^{i-j} x_{i}+p^{i-i_{0}} \beta_{i_{0}} x_{i} e^{1} \\
& =x_{j} \hat{C}-x_{j}+\beta_{i_{0}} x_{i_{0}} e_{1}=x_{j}+k_{j}-x_{j}+t_{1} e_{1} \\
& =t_{1}\left(1-e_{1}\right)+t_{1} e_{1}=t_{1} .
\end{aligned}
$$

To make application of the above Theorem the following is noted.

Proposition. If $p \neq 2$ and if $M$ is a primary Abelian group, then the subring of the endomorphism ring of $M$ generated by the idempotent endomorphisms of $M$ is contained in the subring generated by the automorphisms of $M$.

Proof. If $p \neq 2$ and $e^{2}=e$, then $(e+1)(e-2)=-2$ is a unit. Hence $e+1$ is a unit and each idempotent of $M$ is a sum of two automorphisms of $M$.

The following corollary is now immediate.

Corollary 1. Let $p \neq 2$ be a prime number, $M$ and $N$-primary Abelian groups. Let $A(M)$ and $A(N)$ be the subrings of the endomorphism rings of $M$ and $N$ generated by the automorphisms of $M$ and $N$, respectively. Then any isomorphism of $A(M)$ and $A(N)$ is induced by an isomorphism of $M$ and $N$. 
Remark. Using F. Castagna's example [1, p. 472] and the Proposition above, it is immediate that there are groups for which the subring of the endomorphism generated by the idempotent endomorphisms is properly contained in the endomorphism ring. Thus, while in general this subring may not be the endomorphism ring of $M$, it nevertheless determines $M$.

\section{REFERENCES}

1. F. Castagna, Sums of automorphisms of a primary Abelian group, Pacific J. Math. 27 (1968), 463-473. MR 38 \#5920.

2. I. Kaplansky, Infinite abelian groups, rev. ed., Univ. of Michigan Press, Ann Arbor, Mich., 1969. MR 38 \#2208.

3. R. S. Pierce, Endomorphism rings of primary Abelian groups, Proc. Colloq. Abelian Groups (Tihany, 1963), Akad. Kiadó, Budapest, 1964, pp. 125-137. MR 30 \#3137.

4. - Homomorphisms of primary abelian groups, Proc. Sympos. Topics in Abelian Groups (New Mexico State Univ., 1962), Scott, Foresman, Chicago, Ill., 1963, pp. 215-310. MR 31 \#1299.

5. R. Stringall, Decompositions of Abelian p-groups, Proc. Amer. Math. Soc. 28 (1971), 409-410.

Department of Mathematics, University of California, Davis, California 95616 